\title{
THE EDDY CURRENT DIAGNOSTICS METHOD FOR THE PLASTICALLY DEFORMED AREA SIZES EVALUATION IN NON-MAGNETIC METALS
}

\author{
Artem Yurievich Marchenkov ${ }^{1 *}$, Andrey Alexandrovich Khvostov ${ }^{1}$, Ekaterina Andreevna Slavinskaya ${ }^{1}$, \\ Daria Alexandrovna Zhgut ${ }^{1}$, Dmitriy Vitalievich Chernov ${ }^{2}$ \\ ${ }^{1}$ National Research University Moscow Power Engineering Institute, Moscow, Russian Federation \\ ${ }^{2}$ Mechanical Engineering Research Institute of the Russian Academy of Sciences, Moscow, Russian \\ Federation
}

The aim of this research is to reveal and determine the sizes of plastically deformed areas in metallic non-magnetic materials with the eddy current method. The manuscript contains computational studies to assess the feasibility of using the eddy current method to determine the size of the plastically deformed areas in the metal. The authors developed a two-dimensional mathematical model of the interaction of the electromagnetic field with the control object. The model included poly-harmonic field excitation in a locally deformed plate, and the deformation was modeled in the form of plastically deformed areas under the indents obtained by the ball indentation. The developed model helped to establish the correlation dependencies and linked the informative parameters of the eddy current method with the size of the metal's plastically deformed zone. The authors obtained the calibration curve for copper, the values of which allowed to determine the factual sizes of the plastically deformed area.

Key words: indentation, modeling, stress-strain behavior, the eddy current method, plastically deformed diagnostics, deformations

\section{INTRODUCTION}

One of the main tasks of the technical diagnostics of industrial facilities is to control the stress-strain behavior of the structural material, in particular, to identify areas of plastically deformed with an assessment of their size. The stress-strain behavior (SSB) can be controlled using analytical and computational-experimental methods (see, for example, [1 - 3]). The use of computational and experimental diagnostic methods is necessary to assess the SSB of metal in structures since they consider the real changes in the physicomechanical characteristics of the test object as well as the redistribution of existing loads between its elements over time.

Currently, tensometry is one of the most widely used methods for assessing the SSB in metal. The problems of technical diagnostics, as a rule, use the method of electrotensometry based on a change in the electrical resistance of metals and semiconductors induced by deformations. Despite the indisputable advantages of these methods (high resolution, control efficiency, the ability to measure stresses inducing the object from the inside), their practical implementation is usually difficult due to the high complexity and (in the case of acoustic tensometry) smallness of acoustic effects and the influence of external factors, as well as the complexity of the mathematical description of the propagation of elastic waves in a stressed medium.

The SSB parameters of ferromagnetic objects are assessed using magnetic control methods based on the Villari effect - the phenomenon involving changes in the magnetization of an object induced by mechanical deformations. The most widely used technologies for diagnos- ing plastically deformed zones include methods based on measuring the coercive force, Barkhausen noise, and the magnetic field in the region of local magnetized areas [4]. Alongside this, it should be noted that the characteristics of the material's magnetic properties depend not only on mechanical stresses but also on chemical composition and structure, which, in some cases, complicate diagnosis using the indicated methods.

A promising non-destructive method that allows for the assessment of the SSB of metal of electrically conductive objects is eddy current structuroscopy [5]. Pulse excitation is used to assess the structural-mechanical state of the inner layers of the material. This excitation includes in its spectrum an infinite set of frequencies [6] or polyharmonic excitation [7].

One of the most informative parameters of eddy current monitoring is the applied voltage $U_{a v}$, recorded by the measuring coil of the eddy current transducer (ECT). The applied voltage $U_{a v}$ is used to numerically assess the defectiveness of the controlled item. Many factors influence the value of $U_{a v}$, among which there are both technological control parameters (for example, the distance between the eddy current transducer and the surface of the control object, the geometric parameters of the windings, etc.), and the physical properties of the controlled material (for example, a change in its electrical conductivity). In this regard, when conducting eddy current monitoring with specified parameters, the values of the applied voltage in metal sections with and without defects will differ due to the different conductivity of the studied sections

$U_{a v}=U-U_{0}$ 
where $U_{0}$ is the voltage registered at the defect-free region; $U$ is the voltage recorded during eddy current control.

Eddy currents are not induced with a sufficiently large distance between the sensor and the surface of the control object. As the transducer approaches the surface of the product, eddy currents occur, the circuit of which, as a rule, repeats the shape of the exciting coil. When defects arise and develop, the shape of the eddy current circuits changes, leading to a change in the parameters of the secondary magnetic field. The perturbations of the electromagnetic field are recorded by a measuring coil connected to the data acquisition and processing unit.

It should be noted that the diagnostic signal recorded by the receiving transducer depends not only on the density of eddy currents, the penetration depth of which depends on the physical parameters of the controlled product, but also on the removal of the measuring coil from the control object. The magnitude of the applied voltage can be estimated using analytical models based on the laws of interaction of electromagnetic fields with conductive material and the parameters of the measuring equipment. In the case of an overhead double-winding converter, the value of the applied voltage is calculated according to the formula below:

$$
\begin{aligned}
& \dot{U}_{a v}= \\
& j \mu_{0} W_{e} W_{m} \omega \dot{I} R \pi \int_{0}^{\infty} \underline{\varphi_{O c}} \exp \left(-x h_{*}\right) \times \\
& J_{1}\left(x \frac{R_{e}}{R_{m}}\right) J_{2}\left(x \frac{R_{m}}{R_{e}}\right) d x,
\end{aligned}
$$

where $j=\sqrt{-1}$ the imaginary unit; $\mu_{0}$ is the vacuum permeability; $W_{e}, W_{m}$ is the amount of turns of the excitation and measuring windings respectively;

$R=\sqrt{R_{e} R_{m}}$ is the equivalent radii of ECT; $R_{e}, R_{m}$ are the average radii of the exciting and measuring windings respectively; is the generalized parameter defining the distance between the centers of the ECT's windings and the surface of the controlled object; he, hm are the distance between the centre of the corresponding ECT's winding and the outer surface of the controlled object; $J_{1}$, $\mathrm{J}_{2}$ are the Bessel's functions of the first and second kind respectively; $\varphi_{0 c}$ is the influence function of the controlled object, which depends on the boundary conditions.

A change in the electrical conductivity of metal during its plastic deformation and the presence of unambiguous dependencies between the conductivity and the degree of its deformation allows for using the eddy current method to determine the sizes of plastically deformed areas of metal.

This manuscript contains computational studies to evaluate the feasibility of using the eddy current method to identify the sizes of plastically deformed areas in a deformed metal. A plate of a nonmagnetic material containing a plastically deformed area obtained by pressing an indenter was the object for constructing the model. At present, the strains distribution in a metal under an indent during indentation is rather well studied [8-10], including in studies conducted by the authors [11, 12]. This allows for specifying the shape and size of the plastically deformed area of the metal under the indent in the model, as well as for simulating the change in electrical conductivity in this area and identifying the eddy current control parameters that are most sensitive to changes in its size.

\section{THE METHOD FOR CONDUCTING THE TESTS}

A two-dimensional finite element model of eddy current control was constructed to solve the problem. It is shown in Figure 1.

The model developed in the Ansys software environment consists of an overhead transformer transducer, which is a coaxially arranged excitation and measurement windings with a diameter of $10 \mathrm{~mm}$, and a copper plate with a thickness of $\mathrm{H}=10 \mathrm{~mm}$ with a surface defect in the form of an indent from the ball indenter with a diameter of $D=10 \mathrm{~mm}$. The values of the indents d diameters on the surface of the plate varied from 1 to $5 \mathrm{~mm}$ in increments of $0.5 \mathrm{~mm}$ (see Table 1). The values of the indent depths $t$ for a given indent diameter $d$ and indenter diameter $D$ can be determined by the following formula:

$t=\frac{1}{2}\left(D-\sqrt{D^{2}-d^{2}}\right)$

For a given range of indent diameters, the indent depths calculated by formula (2) lay in the range $t=25$...670 $\mu \mathrm{m}$. The plastically deformed area was modeled as an elliptical region around the indentation, the size of which varies depends on the indentation depth, according to the diagram shown in Figure 2.

Based on the results of studies of the deformation fields propagation under the indents obtained by indentation of a ball indenter [10, 11], the depth of the plastically deformed area propagation was taken to be equal to $T=15 \bullet$. Thus, the size range of the plastically deformed areas in the direction of eddy current control varied from $\mathrm{T}=0.38 \mathrm{~mm}$ (for the indent diameter $\mathrm{d}=0.5 \mathrm{~mm}$ ) to $\mathrm{T}=10 \mathrm{~mm}$ (for the indent diameter $\mathrm{d}=5 \mathrm{~mm}$ ). The val-

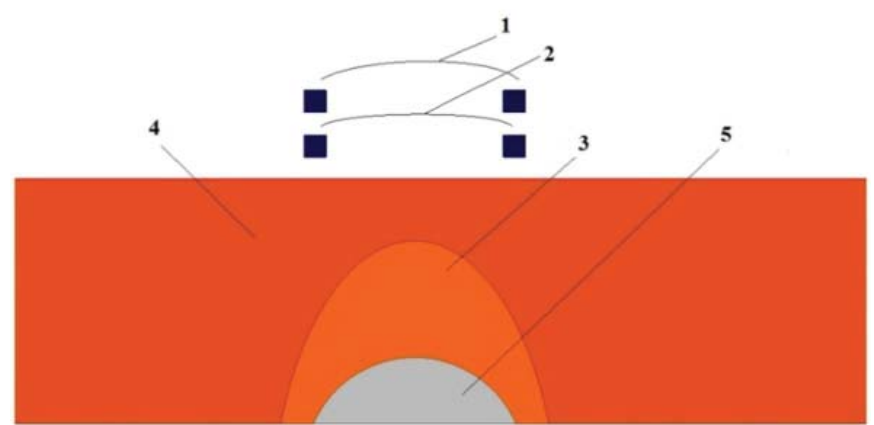

Figure 1: A finite-element two-dimensional model of an eddy current transducer mounted above an indent from a ball indenter with a diameter of $10 \mathrm{~mm}$ in a copper plate:

1 - excitation winding, 2 - measuring winding, 3 - plastically deformed area, 4 - copper plate, 5 - indent 


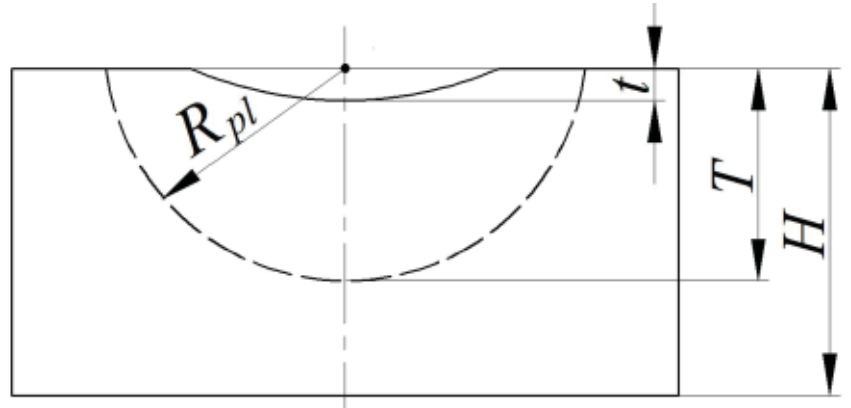

Figure 2: Scheme of the plastically deformed area under the indent obtained by a ball indenter: $t$ is the depth of the indent; $T$ is the depth of plastically deformed area propagation; $R_{p l}$ is the radius of the plastically deformed area; $H$ is the plate thickness

ues of all dimensional parameters of prints and zones of plastic deformation are given in Table 1.

The distribution of deformations in the volume of the metal under the indent is not uniform [8-10] - the values of the intensity of deformations gradually decrease as the distance from the indent deep into the deformed metal. The classical Hill-Johnson scheme [8] was adopted as a diagram of the deformed volume of the indentation metal (Figure 3).

Table 1: The sizes of parameters for indents and the plastically deformed areas set in the model

\begin{tabular}{|c|c|c|c|}
\hline S/N & $\begin{array}{l}\text { Indent } \\
\text { diameter } \\
\mathrm{d}, \mathrm{mm}\end{array}$ & $\begin{array}{l}\text { Indent } \\
\text { depth } \\
\mathrm{t}, \mu \mathrm{m}\end{array}$ & $\begin{array}{l}\text { Depth of the } \\
\text { plastically } \\
\text { deformed area } \\
\text { propagation T, } \mathrm{mm}\end{array}$ \\
\hline 1 & 1.0 & 25 & 0.38 \\
\hline 2 & 1.5 & 57 & 0.86 \\
\hline 3 & 2.0 & 101 & 1.52 \\
\hline 4 & 2.5 & 159 & 2.39 \\
\hline 5 & 3.0 & 230 & 3.45 \\
\hline 6 & 3.5 & 316 & 4.74 \\
\hline 7 & 4.0 & 417 & 6.26 \\
\hline 8 & 4.5 & 535 & 8.03 \\
\hline 9 & 5.0 & 670 & 10.00 \\
\hline
\end{tabular}

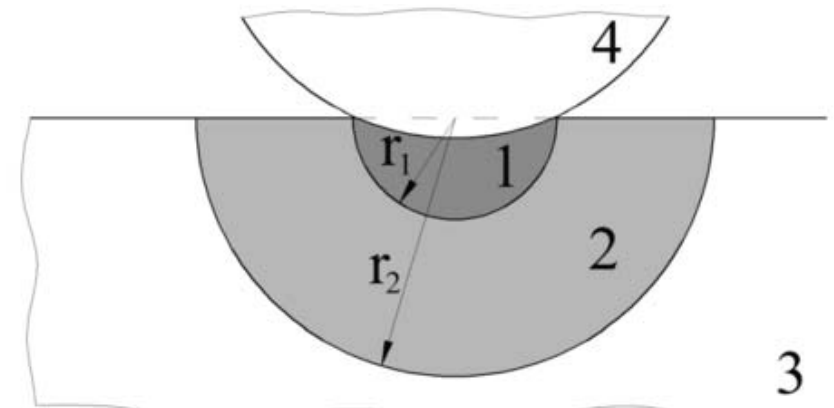

Figure 3: The Hill-Johnson model of the plastically deformed propagation under the indent obtained by a ball indenter: 1 is the hydrostatic core of radius $r_{1} ; 2$ is elastic-plastic strain area of radius $r_{2} ; 3$ is the elastic deformation area; 4 is ball indenter
In the Hill-Johnson model, the stressed metal under the indent is conventionally divided into two main areas - the hydrostatic core area (item 1 in Figure 3 ) and the elastic-plastic strain area (item 2 in Figure 3). It is assumed that the main processes of stress occur in the region of the hydrostatic core, and the maximum stress remains constant with increasing depth of indenter penetration. Based on [8, 13], the authors assume that for copper the average value of the degree of deformation in the hydrostatic core area is on average about $30 \%$, and, in the elastic-plastic strain area, about $8 \%$.

The value of the electrical conductivity of undeformed technically pure copper was taken to be equal to $\sigma_{\mathrm{m}}=58 \mathrm{MS} / \mathrm{m}$. The average value of the electrical conductivity of a plastically deformed metal with a deformation degree of $\Psi=8 \%$ based on reference data for technically pure copper was taken to be $\sigma_{\mathrm{cd} 1}=57.5 \mathrm{MS} / \mathrm{m}$, for a metal with a degree of stress of $\Psi=30 \%$, the conductivity $\sigma_{\mathrm{cd} 2}=57.2 \mathrm{MS} / \mathrm{m}$.

In order to localize the electromagnetic field in the simulated area, boundary conditions were used, according to which the magnetic induction vector is parallel to the surfaces of the model sample. An electromagnetic wave was excited using a double-winding sensor overhead located above the surface of a conductive product. The model used polyharmonic excitation, which allows for layer-by-layer control of the conductivity of a deformed product. For a copper plate with a thickness of $10 \mathrm{~mm}$, the frequencies of poly-harmonic excitation were chosen so that the penetration depth corresponded to $\delta=1,2, \ldots 10 \mathrm{~mm}$. A sinusoidal current with a frequency $\mathrm{f}$ in the range from 45 to $4500 \mathrm{~Hz}$ with a step of $45 \mathrm{~Hz}$ and an amplitude of $1 \mathrm{~A}$ was supplied to the field winding. The choice of the frequency range $f$ is due to the calculation of the penetration depth of a plane electromagnetic wave into an electrically conductive medium, which corresponds to the penetration depth of the eddy current field $\delta$ in a controlled object. The penetration depth $\delta$ is inversely proportional to the square root of the excitation frequency $f$, subject to the homogeneity of the electrophysical properties of the object - relative magnetic permeability and conductivity. Thus, the generation of high-frequency currents is necessary to control the surface layers, while the low-frequency mode ensures the penetration of eddy currents into the inner layers of the object. The calculated frequency range corresponds to layer-by-layer scanning of an object by recording the applied voltage $U_{a v}$ at each frequency using a measuring winding.

As a result of the study, the change in the applied voltage $U_{a v}$ from the size of the plastically deformed area was evaluated. The amplitude of $\left(\left|U_{a v}\right|\right)$ and phase $(\varphi)$ define the value of $U_{a v}$. The transition to the plastically deformed area is characterized by a change in the electrical conductivity relative to the main volume of the controlled material, which will affect both the amplitude and the phase of the applied voltage. Therefore, the task of identifying the plastically deformed area by the eddy current method 


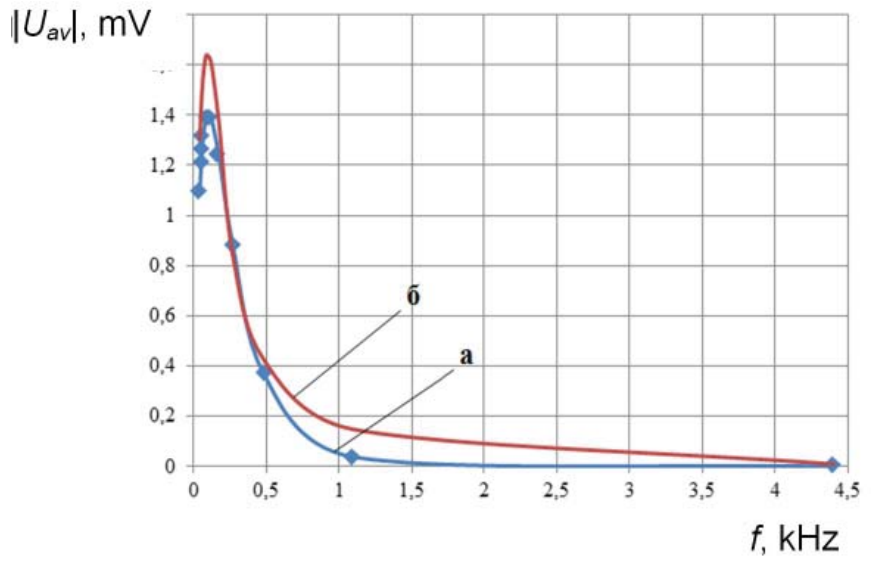

Figure 4: Correlation of applied voltage $U_{a v}$ amplitude with the excitation frequency $f$ in the absence of the plastically deformed area (1) and the presence of the plastically deformed area from the imprint $d=3 \mathrm{~mm}$ (2) is reduced to the selection of such control conditions that will provide maximum sensitivity to changes in electrical conductivity in the subsurface layer. Using a technique based on multi-frequency excitation, it becomes possible to obtain information about the distribution of the conductivity value over the thickness of the test object, thereby determining the size of the plastically deformed area.

\section{RESULTS AND DISCUSSION}

The primary result of building a finite element model are the dependencies of the applied voltage $\left|U_{a v}\right|$ amplitudes on the excitation frequency of the electromagnetic signal $f$. Figure 4 demonstrates the influence of the stressed area conductivity on the eddy current test results. Figure 4 shows dependencies of $\left|U_{a v}\right|$ on $f$ for the studied plate under the absence of the plastically deformed area (curve 1), as well as for the plate with the residual indent with the diameter of $\mathrm{d}=3 \mathrm{~mm}$ (curve 2).

Figure 4 shows that the change in metal conductivity in the plastically deformed area significantly influences the results of the eddy current tests. The frequency range of $f \geq 0.5 \mathrm{kHz}$ displays the largest difference in the values of the applied voltage amplitude. Thus, at $f=1 \mathrm{kHz}$, the value of $U_{a v}$ for the plate with the indent is around $0.16 \mathrm{mV}$, while for the non-stressed metal, it does not exceed $0.03 \mathrm{mV}$. The frequencies range of $0.2 \leq f<1 \mathrm{kHz}$ demonstrate a sharp increase in the applied voltage. The frequencies of $f \leq 0.2 \mathrm{kHz}$ also demonstrate the difference in the signal of $U_{a v}$ from stressed and non-stressed metals.

A slight decrease in conductivity, which appears at plastically deformed of the metal, results in the changes of the parameters of the electromagnetic signals, which were registered at eddy current test with poly-harmonic excitation. The most intense change in the applied voltage amplitude is registered at such frequencies $f$ where the depth of eddy currents contours' propagation approximately correlates with the size of the plastically deformed area. A decrease in the excitation frequency of

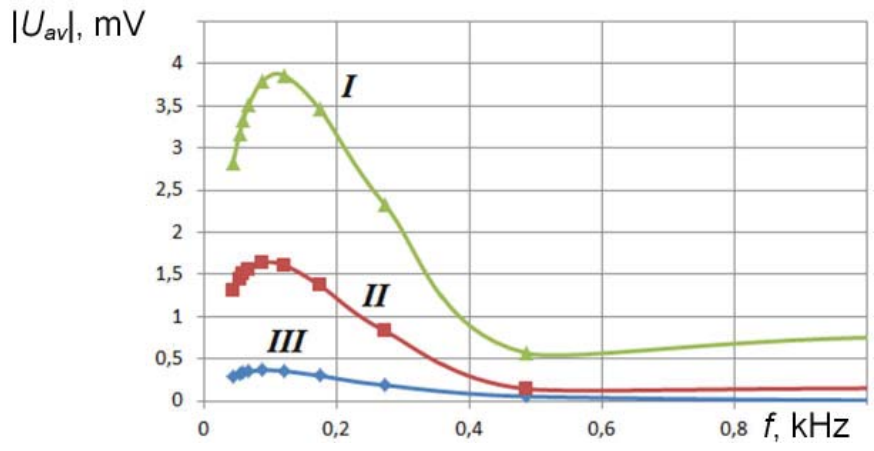

Figure 5. The results of plotting the applied voltage amplitude $|u a v|$ on the excitation frequency of the electromagnetic signal $f$ with the presence of the plastically deformed area from indent $d=1 \mathrm{~mm}(\mathrm{I})$,

$$
d=3 \mathrm{~mm} \text { (II) and } d=5 \mathrm{~mm} \text { (III) }
$$

the electromagnetic wave leads to the increased depth of propagation, ad thus to the increased sensitivity of the eddy current test to the changes in the indent's size. As Figure 4 shows, the graphs of $\left|U_{a v}\right|(f)$ have a specific peak, registered at the propagation depth of $\delta$, comparable to the thickness of the tested object. Thus, in order to determine the size of the plastically deformed area by the eddy current method, it is necessary to analyze the slope of the characteristic $\left|U_{a v}\right|(f)$ in the frequency range from 0.2 to $1 \mathrm{kHz}$.

A finite element model of the interaction of the eddy current field with a stress metal at various sizes of the indent was developed to solve this problem. According to the results of mathematical modeling, the dependencies $\left|U_{a v}\right|$ on frequency $f$ for different sizes of plastically deformed areas. Figure 5 shows the appearance of the obtained dependencies for some sizes of the plastically deformed areas.

Figure 5 shows the dependencies of $\left|U_{a v}\right|(f)$, registered at the presence of indents of various sizes from the penetration of the ball indenter. The forms of the resulted dependencies are similar to each other and correspond to the Gauss function of the second order. The value of the applied voltage amplitude is close to zero at high-frequency excitation of the electromagnetic wave. Reducing the frequency $f$ results in increased values of $\left|U_{a v}\right|$. It should be noted that the increase rate of the applied voltage amplitude correlates with the indent's size. In order to calculate the factual size of the plastically deformed area, the following coefficients of the approximation function should be used:

$\left|u_{a v}\right|(f)=a_{1} e^{\left(-\left(\frac{f-b_{1}}{c_{1}}\right)^{2}\right)}+a_{2} e^{\left(-\left(\frac{f-b_{2}}{c_{2}}\right)^{2}\right)}$

where $a_{1}, b_{1}, c_{1}, a_{2}, b_{2}, c_{2}$ are the coefficients of the approximation function.

Processing of the dependencies shown in Figure 5 results in the findings that the most informative parameter of the eddy current control, which is sensitive to the presence of the plastically deformed area in the metal, is the value of the criterial parameter $k_{c}=a_{1} / a_{2}$. The graphical 
dependency of the parameter kc on the size of the plastically deformed area is shown in Figure 6.

Regression analysis of the obtained dependence of the criteria parameter $\mathrm{k}_{\mathrm{c}}$ on the size of the plastically deformed area, which is shown in Figure 6, demonstrated that exponential function can display it with sufficient accuracy.

$$
k_{c}\left(\frac{T}{H}\right)=2.53 \cdot\left(\frac{T}{H}\right)^{6.87}+0.27
$$

where $\mathrm{T}$ is the depth of plastically deformed area propagation, $\mathrm{H}$ is the plate's thickness.

The calibration curve shown in Figure 6 can be divided into two sections. The first section corresponds to the range $\mathrm{T} / \mathrm{H}<0.85$, which demonstrates a smooth change in the values of the criterial parameter depending on the size of the plastically deformed area. The subsequent increase in the $\mathrm{T} / \mathrm{H}$ parameter results in the sharp increase in $\mathrm{k}_{\mathrm{c}}$. This effect can be connected to the changes in the metal's conductivity at the near-surface layers, which sufficiently increases the value of $\left|U_{a v}\right|$ for the high-frequency components of the eddy current signal.

Thus, the criterial parameter kc is highly sensitive to the changes of the relative depth of the plastically deformed area propagation $\mathrm{T} / \mathrm{H}$. This means that the mentioned parameter can be used to characterize the presence of the plastically deformed areas in the non-magnetic metal objects with the specified thickness.

\section{CONCLUSION}

The manuscript considers the problem of assessing the sizes of the plastically deformed areas on metals by the eddy currents method. The authors developed the two-dimension model of the eddy current transducer, which includes poly-harmonic electromagnetic field excitation in the deformed copper plate.

The authors defined that in order to determine the factual size of the plastically deformed area by the eddy current method, the values of the applied voltage amplitude should be registered. At that, using the multi-frequency approach allows for the layerwise scanning of the state

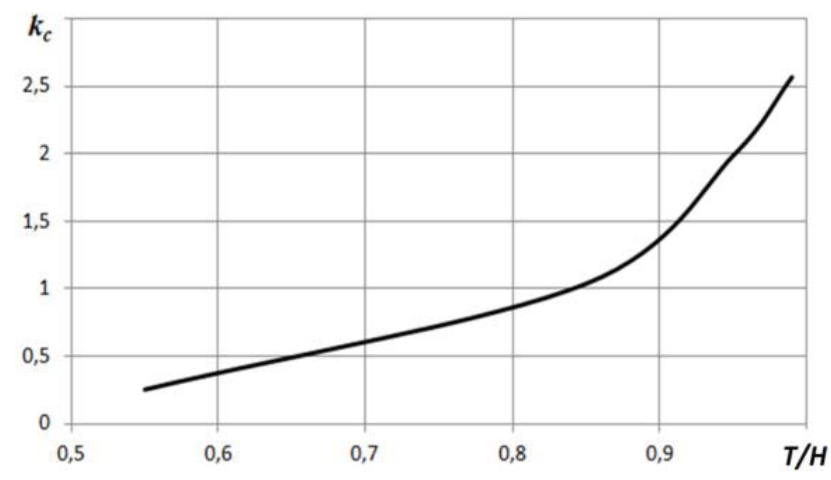

Figure 6: Dependence of the criteria parameter $k_{c}$ on the relative depth of the plastically deformed area propagation $\mathrm{T} / \mathrm{H}$ of the item exposed to the plastically deformed. The approximating Gauss function of the second-order allowed for determining the most informative parameter , which correlates with the values of the plastically deformed area depth.

The authors obtained the calibration curve for copper, the values of which allow for determining the factual sizes of the plastically deformed area.

The approach for the assessing the plastically deformed area size using the eddy current method allows for obtaining the information on the factual state of the metal. The method of poly-harmonic eddy currents excitation allowed for determining the correlation dependencies between the gradient in conductance and the strain degree.

The analytical models for assessing the state of the industrial facilities can be built based on the identified regularities and calibration curves. This also includes the possibility of determining the inner strains and forecasting of the residual lifetime.

\section{ACKNOWLEDGEMENTS}

The manuscript is written in "NRU "MPEl" under the grant of the President of the Russian Federation for the state support of young Russian scientists - candidate of sciences (agreement No. 075-15-2019-333 (MK2162.2019.8)).

\section{REFERENCES}

1. Jia, L.J., Ge, H. (2019) Stress-strain behaviors in large plastic strain ranges under monotonic loading, Springer Tracts in Civil Engineering, 13-23.

2. Bhandari, R., Biswas, P., Mondal, M.K., Mandal, D. (2018). Finite element analysis of stress-strain localization and distribution in $\mathrm{Al}-4.5 \mathrm{Cu}-2 \mathrm{Mg}$ alloy, Transactions of Nonferrous Metals Society of China (English Edition), vol. 28, no. 6, 1200-1215.

3. Nikolaevich, S. A., Vladimirovich, E. O., Valeryevich, B. A., \& Prokic, M. [2016]. Stress intensity factor in welded joints out of double angles in lattice steel structures. Journal of Applied Engineering Science, 14(2), 285-289.

4. Yao, K., Wu, L.B., Wang, Y.S. (2019). Nondestructive Evaluation of Contact Damage of Ferromagnetic Materials Based on Metal Magnetic Memory Method, Experimental Techniques, vol. 43, no. 3, 273-285.

5. Habibalahi, A., Habibalahi, M., Samadian, K. (2018). Pulsed eddy current applied to measure residual stress in welding, Journal of Testing and Evaluation, vol. 46, no. 6, 20160504.

6. Lunin, V.P., Chernov, L.A., Slavinskaya, E.A., Terekhin, I.V., Barat, V.A. (2016). The method of controlling the changes in electrical conductivity of the inner layers of non-magnetic metals and the way of its performing. The RF Patent No. 2597960. Bulletin 2016. No. 26 
7. Svatos, J, Pospisil, T., Vedral, J. (2018). Application of poly-harmonic signals to eddy-current metal detectors and to advanced classification of metals, Metrology and measurement systems, vol. 25, no. 2, $387-402$.

8. Breumier, S., Villani, A., Maurice, C., Lévesque, M., Kermouche, G. (2019). Effect of crystal orientation on indentation-induced residual stress field: Simulation and experimental validation, Materials and Design, vol. 169, no. 5, 107659.

9. Pero, R., Maizza, G., De Marco, F., De Fazio, P., Montanari, R, Ohmura, T. (2019). Determination of the yield radius and yield stress in 2198-T3 aluminum alloy by means of the dual-scale instrumented indentation test, Materials Transactions, vol. 60, no. 8, 1450-1456.

10. Chaudhri, M.M. (1996). Subsurface plastic strain distribution around spherical indentations in metals, Philosophical Magazine A: Physics of Condensed Matter, Structure, Defects and Mechanical Properties, vol. 74, no. 5, 1213-1224.
11. Matyunin, V.M., Marchenkov, A.Yu., Terent'ev E.V., Demidov, A.N. (2016). Substantiation of the ratio of the sample thickness to the indentation depth in hardness measurements, Russian metallurgy (Metally), no. 13, 47-50.

12. Matyunin, V.M., Kazantsev, A.G., Marchenkov, A.Yu. (2018). Distribution of stresses and deformations in the deformed metal volume at indenting of a spherical indenter, Inorganic materials, vol. 54, no. 15 , 4954.

13. Chaudhri, M.M. (1998). Subsurface strain distribution around Vickers hardness indentations in annealed polycrystalline copper, Scripta Materialia, vol. 46, no. 9, 3047-3056. 